\title{
Xenon out of its shell
}

Like all noble gases, xenon is colourless, odourless and inflammable - but it is also more reactive, and much rarer, than its lighter relatives. Ivan Dmochowski ponders how xenon, though initially slow to earn a spot in the periodic table, is now at the forefront of advances in science and technology.

F or all their nobility, elements of group 18

have lived in relative obscurity. In

1869, Mendeleev's first periodic table did not include them, as closed-shell elements remained undiscovered until Lord Rayleigh and William Ramsey isolated argon in 1894. Remarkably, Ramsey then went on to isolate helium (1895) and radon (1908) and, with Morris Travers, to discover krypton, neon and xenon (1898). It was debated whether the 'inert' monoatomic gases belonged to the periodic table owing to their lack of 'chemical properties'; elements coming late to the party, with no unpaired electrons to share, may not get a seat at the table. But Ramsey established their position between halogens and alkali metals, and was awarded the Nobel Prize in Chemistry in 1904 for these elemental contributions.

Xenon's discovery concluded an intense period of research on noble gases. Much heavier than neon and krypton, it had not been explicitly predicted, and was sufficiently rare to avoid chance detection. Its discovery was almost accidental. Travers had spent months fractionating liquid air residues, but having isolated the lighter noble gases he would typically ignore any additional volatile components. On one occasion, however, he collected the gas remaining in his apparatus. The residue (just $0.3 \mathrm{ml}$ ), introduced into a spectrum tube, revealed a brilliant blue colour characteristic of xenon. Its rarity in air $\left(0.09 \mathrm{ml} \mathrm{m}^{-3}\right)$ combined with high density $\left(5.8 \mathrm{~g} \mathrm{l}^{-1}\right)$ violated common experience, and so xenon was named after $\xi \dot{\varepsilon} v o v$, a Greek word meaning 'foreign'.

Enshrouded by five filled electron shells (electronic configuration [Kr] $5 s^{2} 4 d^{10} 5 p^{6}$ ), xenon has been slow to reveal its secrets. Its scarcity in the Earth's atmosphere 10 - to 100,000 -fold rarer than the lighter noble gases, despite their lower escape

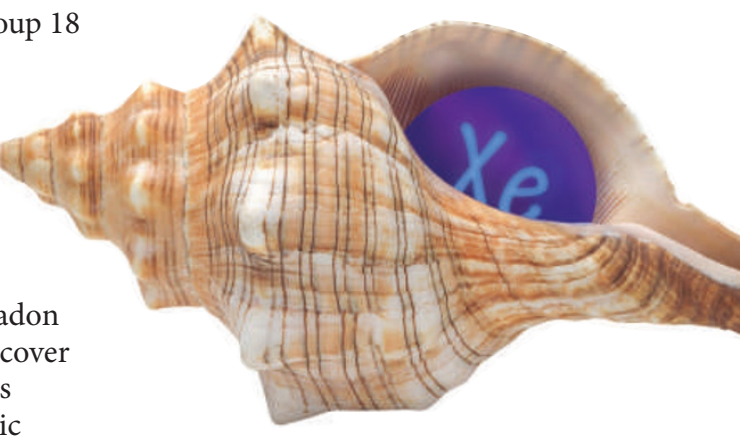

Xenon, initially deemed unreactive, is now finding ways to come out of its shell.

velocities - has been a long-standing mystery. Recent X-ray-diffraction studies now indicate that xenon can substitute for silicon in quartz at high pressure and temperature, suggesting that the Earth's 'missing xenon' may be covalently bonded to oxygen in the continental crust.

Kossel and Pauling (in 1916 and 1932, respectively) predicted that xenon's ionization potential $(12.1 \mathrm{eV})$ should allow its reaction with strong oxidants. This was finally confirmed in 1962, when Neil Bartlett observed that xenon reacted with vapours of platinum hexafluoride $\left(\mathrm{PtF}_{6}\right)$ to form a yellow-orange solid compound - a discovery widely recognized as one of the most significant inorganic chemistry advances of the twentieth century. The syntheses of various fluorides, oxides and perxenates $\left(\mathrm{XeO}_{6}{ }^{4-}\right.$ salts $)$ quickly followed. Xenon can form covalent bonds to carbon and nitrogen, and, as shown by the recent synthesis of a compound with gold $\left(\mathrm{AuXe}_{4}{ }^{2+}\right)$, also coordinates metal ions.

Xenon now finds uses in fields as varied as lasers and incandescent lamps, plasma display panels, silicon etching in semiconductor manufacturing and medicine. In 2008, twelve million litres of xenon were extracted from the atmosphere, and production is growing to meet technological needs.
Xenon's polarizability (about 4 compared with 0.2 for $\mathrm{He}$ ) contributes to its affinity for hydrophobic cavities in proteins, which is relevant not only for protein crystallography but also for anaesthetic use. Behnke deduced

that xenon was an anaesthetic in 1939, after observing 'drunkenness' in deep-sea divers, and it was first used for surgical purposes in 1951. It has gained newfound popularity, based on its non-toxicity and low environmental impact (compared with halocarbons), and a xenon-based anaesthetic (LENOXe) was commercialized in 2007.

Xenon has more than 50 isotopes, including nine stable ones (second only to tin, which has ten). ${ }^{129} \mathrm{Xe}$, with a spin- $1 / 2$ nucleus, provides large NMR signals for imaging studies in the lungs. Moreover, the ${ }^{129} \mathrm{Xe}$ NMR chemical shift is extremely sensitive to stereo-electronic perturbations of the ${ }^{129} \mathrm{Xe}$ atom, and xenon biosensors based on these phenomena are now under development.

One of the most exciting new applications is in xenon-ion propulsion engines for space travel. Onboard NASA's Dawn spacecraft, launched in 2007 to study distant asteroids Vesta and Ceres, cationic xenon atoms are accelerated towards a negatively charged grid, with exhaust speeds of approximately one million $\mathrm{km} \mathrm{h}^{-1}$. The thrust generated by xenon is only 0.1 newtons, roughly the weight of a piece of paper, but can gradually (over many months) increase the velocity of the spacecraft by $15,000 \mathrm{~km} \mathrm{~h}^{-1}$. This reduces the requirement for much heavier chemical fuel, thereby lowering launch cost, while increasing travel range. Whether through oxidation, coordination or ionization, xenon has revealed properties that lead to extremely versatile applications - and this field is still in its infancy.

IVAN DMOCHOWSKI is in the Department of Chemistry at the University of Pennsylvania, US.

e-mail: ivandmo@sas.upenn.edu

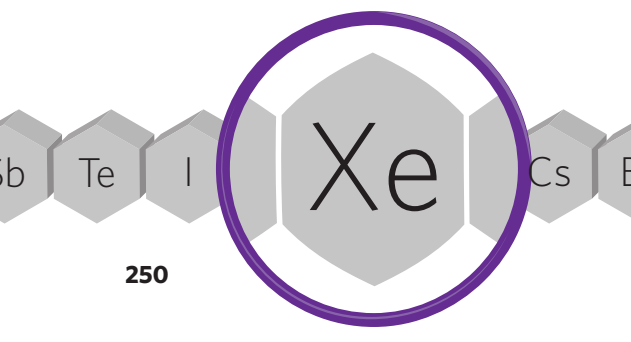

